\title{
Einfluß von Fehlstellen und Verunreinigungen auf den Ordnungs-Unordnungs-Übergang im hexagonalen Eis Ih
}

\author{
I. Sesselmann und D. Helmreich \\ Physik-Department, Technische Universität München \\ (Z. Naturforsch. 26 a, $803-809$ [1971] ; eingegangen am 9. Februar 1971)
}

Herrn Professor Dr. Nikolaus Riehl zum 70. Geburtstag gewidmet

\begin{abstract}
Die Temperaturabhängigkeit der longitudinalen elastischen Konstanten $c_{33}$ von reinem und $c_{33 H F}$ von $\mathrm{HF}$-dotiertem schweren Eis wurde im Bereich zwischen 80 und $200^{\circ} \mathrm{K}$ mit einer Phasenvergleichstechnik gemessen. Beim HF-dotierten $\mathrm{D}_{2} \mathrm{O}$-Eis wurde unterhalb $150{ }^{\circ} \mathrm{K}$ eine positive Anomalie gefunden. Reines $\mathrm{D}_{2} \mathrm{O}$-Eis verhält sich im gemessenen Temperaturbereich normal.

Die Ergebnisse können erklärt werden durch mikroskopische, im Hochtemperaturbereich stabile Keime der Tieftemperaturphase, die beim Abkühlen Zentren der neuen Phase werden.
\end{abstract}

\section{Einleitung}

Das uns wohlvertraute hexagonale Eis Ih ist nur eine von den vielen möglichen Formen festen Wassers. Heute sind neun weitere Eismodifikationen bekannt: außer dem kubischen Eis Ic, das ebenfalls bei Atmosphärendruck beständig ist, gibt es die Hochdruckmodifikationen Eis II - IX. Eine Beschreibung der einzelnen Modifikationen ist in ${ }^{1-4} \mathrm{zu}$ finden.

Zwei Dinge sind diesen Eismodifikationen gemeinsam: Die Bausteine sind Wassermoleküle, die bei der Bildung einer dieser Modifikationen nicht zerstört werden. Die Wassermoleküle sind in einem tetraedrischen Gitter angeordnet, ihre molekulare Achse hat dadurch sechs Einstellmöglichkeiten - es besteht also die Möglichkeit einer Orientierungsunordnung.

Abgesehen von der metastabilen Phase IV gibt es zu den Hochdruckmodifikationen eine Tieftemperaturphase, bei der das Protonengitter geordnet ist ${ }^{2,3,5}$. Eis IX ist die geordnete Tieftemperaturphase zu Eis III ${ }^{6}$, Eis VIII die zu Eis VII ${ }^{7}$. Für

Sonderdruckanforderungen an Dr. D. Helmreich, PhysikDepartment der Technischen Universität München, $D-8000$ München 2, Arcisstraße 21.

1 H. Gränicher, in: Physics of Ice (N. Riehl, B. Bullemer u. H. Engelhardt, Ed.), Plenum Press, New York 1969, S. 1.

2 E. Whalley, in: Physics of Ice (1. c. ${ }^{1}$ ), S. 19.

3 J. W. Glen, Sci. Prog. Oxford 57, 1 [1969].

4 B. Kамв, in: Structural Chemistry and Molecular Biology (A. Rich u. N. Davidson, Ed.), Freeman and Co., San Francisco 1968.

5 W. C. Hamilton, B. Kamb, S. J. La Placa u. A. Prakash, in: Physics of Ice, S. 44.

6 E. Whalley, J. R. B. Heath u. D. W. Davidson, J. Chem. Phys. 48, 2362 [1968].
Eis V und VI gibt es Hinweise, daß sich bei tieferen Temperaturen eine geordnete Protonenstruktur einstellt ${ }^{2,5}$. Eis II ist in seinem Existenzbereich geordnet $^{8}$.

Eine Vielzahl von Untersuchungen an hexagonalem Eis Ih brachte ebenfalls Hinweise auf eine Tieftemperaturphase mit geordneter Protonenstruktur. Die verschiedenen Messungen, wie Untersuchungen der statischen $\mathrm{DK}^{9}$, der spezifischen Wärme ${ }^{10-12}$, der Doppelbrechung ${ }^{13}$, von Polarisations-Glow-Kurven ${ }^{14-16}$, der elastischen Konstanten ${ }^{17}$ und der Neutronenstreuung ${ }^{18}$, ergaben folgendes Bild: Bei nicht zu reinen Eiskristallen treten in einem größeren Temperaturbereich $\left(80-150^{\circ} \mathrm{K}\right)$ anomale Änderungen der Meßwerte auf. Durch zusätzliches Dotieren (meist wird HF verwendet) kann dieser Effekt vergrößert und $\mathrm{zu}$ höheren Temperaturen verschoben werden.

Die gefundenen Anomalien machen auch beim hexagonalen Eis einen Ordnungs-Unordnungs-Übergang in der Protonenstruktur sehr wahrscheinlich. Systematische Untersuchungen der Sauerstoffstruktur in diesem Temperaturbereich stehen zwar noch

7 E. Whalley, D. W. Davidson u. J. R. B. Heath, J. Chem. Phys. 45, 3976 [1966].

8 B. Камв, Acta Cryst. 17, 1437 [1964].

9 O. Dengel, U. Eckener, H. Plitz u. N. Riehl, Phys. Letters 9, 291 [1964].

10 N. F. Giauque u. J. W. Stout, J. Amer. Chem. Soc. 58, 1144 [1936].

11 M. A. PICK, in: Physics of Ice, S. 344.

12 A. van den Beukel, Phys. Stat. Sol. 28, 565 [1968].

13 M. Hotellier, Diplomarbeit, Universität Paris 1961.

14 T. Nedetzka, private Mitteilung.

15 P. G. Bishop u. J. W. Glen, in: Physics of Ice, S. 492.

16 H. P. Glockmann, in: Physics of Ice, S. 502.

17 D. Helmreich, in: Physics of Ice, S. 231.

18 R. G. Whiтlocк, M. Sc. Thesis, Univ. Birmingham 1970. 
aus, die bisher durchgeführten Röntgenstrukturuntersuchungen ${ }^{19,} 20$ sprechen jedoch sehr dafür, daß sich die Gitterkonstanten (des Sauerstoffgitters) zwischen Schmelzpunkt und Stickstofftemperatur nicht anomal verhalten. In den Kristall eingelagerte Verunreinigungen haben eine katalytische Wirkung auf den Ordnungs-Unordnungs-Übergang. Der in 21 diskutierte Fall der homogenen Nukleation von geordneten Keimen innerhalb der ungeordneten Hochtemperaturphase wird daher nur eine sehr untergeordnete Rolle spielen. Wie die Messungen zeigen, findet hauptsächlich heterogene Nukleation statt, d. h., die Keime bilden sich vornehmlich an Fehlstellen und Verunreinigungen. Wie in Abschnitt II gezeigt wird, hängt die Nukleationsrate auch noch ab von der Ubertrittswahrscheinlichkeit eines Teilchens aus der ungeordneten $\mathrm{Phase}$ in die geordnete. $\mathrm{H}_{2} \mathrm{O}$. Eis und $\mathrm{D}_{2} \mathrm{O}$-Eis können also ohne weiteres verschiedene Verhaltensweisen zeigen. Die bisher durchgeführten Messungen an reinem $\mathrm{D}_{2} \mathrm{O}$-Eis (spezifische Wärme ${ }^{22}$, elastische Konstanten ${ }^{23,24}$ und Neutronenstreuung ${ }^{18}$ ) verliefen negativ. Die im Rahmen dieser Arbeit durchgeführten Ultraschall-Untersuchungen an reinem und HF-dotiertem $\mathrm{D}_{2} \mathrm{O}$-Eis sollen weitere Aufschlüsse über den Mechanismus des Ordnungs-Unordnungs-Übergangs von Eis Ih liefern.

\section{Theorie der Phasenübergänge}

Phasenübergänge gehen im allgemeinen durch Nukleation von Keimen vor sich, die spontan weiterwachsen, sobald sie eine kritische Größe erreicht haben. Die Nukleationsrate hängt stark von der Unterkühlung ab; die Temperatur, bei $\operatorname{der} \alpha$ - und $\beta$-Phase im Gleichgewicht sind, muß um einen kritischen Wert unterschritten werden (kritische Unterkühlung), damit die Phasenumwandlung eingeleitet wird. Der $\beta$-Keim wird durch eine Folge von monomolekularen Prozessen aus der ursprünglichen $\alpha$ Phase gebildet. Diese Nukleation, bei der die $\beta$-Keime im Innern einer perfekten $\alpha$-Matrix (ohne Strukturfehler und Verunreinigungen) gebildet wer-

19 K. Lonsdale, Proc. Roy. Soc. London A 247, 424 [1958].

20 R. Brill u. A. Tippe, Acta Cryst. 23, 343 [1967].

21 D. Turnbull, Solid State Physics, Vol. 3 [1956].

22 E. A. Long u. J. D. Kemp, J. Amer. Chem. Soc. 58, 1829 [1936].

23 U. Mitzdorf, Diplomarbeit, Techn. Universität München 1969. den, heißt „homogene Nukleation“. Fehlstellen wirken als Keimzentren für die sogenannte "heterogene Nukleation“. Beim weiteren Wachstum der durch homogene oder heterogene Nukleation entstandenen kritischen Keime spielt die Diffusion eine entscheidende Rolle.

Erste Ansätze zu dieser Theorie der Phasenumwandlung stammen von Gibbs. Eine detailliertere Anwendung dieser Ideen auf den Nukleationsprozeß beschreiben VOLMER und Weber, deren Theorie später von BECKER und DöRING erweitert wurde. Neuere Übersichtsartikel schrieben Turnbull ${ }^{21}$, Hirth-Pound ${ }^{25}$ (siehe auch die Monographie von $\left.\mathrm{F}_{\text {INE }}{ }^{26}\right)$. Hier sollen nur einige Ergebnisse der $\mathrm{Nu}$ kleationskinetik und des Wachstums der überkritischen Keime gebracht werden.

\section{Homogene Nukleation}

Bei der Bildung eines $\beta$-Keims durch homogene Nukleation wird die Gibbssche freie Energie des Systems um den Betrag $\Delta G$ geändert (eine evtl. Volumenänderung beim Übergang von $\operatorname{der} \alpha$-Phase in $\operatorname{die} \beta$-Phase wird vernachlässigt) .

$$
\Delta G=a \sigma_{\alpha \beta} i^{2 / 3}+\Delta G_{\mathrm{v}} i v
$$

mit

$$
\Delta G_{v}=G_{\beta}-G_{\alpha},
$$

$G_{a}, G_{\beta}=$ freie Energie der $\alpha-, \beta$-Phase pro Volumeneinheit, $i=$ Anzahl der Moleküle im $\beta$-Keim, $v=$ Volumen eines $\beta$-Moleküls, $\quad a=$ Oberfläche der $\beta$-Keime, $\sigma_{\alpha \beta}=$ Oberflächenspannung zwischen $\alpha$ - und $\beta$-Phase.

Wenn sich die Form des als kugelförmig angenommenen Keims bei weiterem Wachstum nicht ändert, geht die freie Energie $\Delta G$ über ein Maximum mit den Koordinaten

$$
\begin{aligned}
\Delta G^{*} & =4\left(a \sigma_{\alpha \beta}\right)^{3} / 27\left(v \Delta G_{\mathrm{v}}\right)^{2}, \\
i^{*} & =\left(2 a \sigma_{\alpha \beta} / 3 v \Delta G_{\mathrm{v}}\right)^{3} .
\end{aligned}
$$

$i^{*}$ ist die Anzahl der Moleküle in einem kritischen Keim (beim Gefrieren von Wasser bei einer Unterkühlung von $-40{ }^{\circ} \mathrm{C}$ beträgt $i^{*}$ etwa 190 Moleküle ${ }^{27}$ ).

24 U. Mitzdorf u. D. Helmreich, J. Acoust. Soc. Amer., erscheint demnächst.

25 J. P. Hirth u. G. M. Pound, Prog. Materials Sci. 11, 88 [1963].

26 M. E. Fine, Phase Transformations in Condensed Systems, McMillan Comp., New York 1964.

27 N. H. FletCher, The Chemical Physics of Ice, University Press, Cambridge 1970. 
Die Nukleationsrate $I$ ergibt sich zu

$$
I=K_{v}{ }^{\prime} v \exp \left\{-\Delta G^{*} / k T\right\} .
$$

Dabei ist $\quad K_{v}{ }^{\prime}=n^{*} n\left(\sigma_{\alpha \beta} a / 9 \pi k T\right)^{1 / 2}$

und

$$
\boldsymbol{v}=\boldsymbol{v}_{0} \exp \left\{-\Delta G_{\mathrm{a}} / k T\right\}
$$

die mittlere Frequenz, mit der ein Molekül die Phasengrenze $\alpha-\beta$ überschreitet.

$n^{*}=$ Anzahl der Moleküle an der Oberfläche des Keims, $n=$ Anzahl der Moleküle pro Volumeneinheit in der $\alpha$-Phase, $v_{0}=k \Theta / h, \Theta=$ Debye-Temperatur, $\Delta G_{\mathrm{a}}=\mathrm{Ak}$ tivierungsenergie für Volumendiffusion.

Wegen des Zusammenhangs zwischen $\Delta G_{\mathrm{v}}$ und der Unterkühlung $\Delta T^{-}=T_{\mathrm{c}}-T\left(T_{\mathrm{c}}=\right.$ Übergangstemperatur)

$$
\Delta G_{\mathrm{v}}=\Delta S_{\mathrm{v}} T_{\mathrm{c}}-\Delta S_{\mathrm{v}} T=\Delta S_{\mathrm{v}} \Delta T^{-}
$$

kann die Nukleationsrate auch als Funktion der Unterkühlung betrachtet werden. $\Delta S_{\mathrm{v}}$ wird dabei als unabhängig von der Temperatur angenommen.

\section{Heterogene Nukleation}

Strukturfehler und Verunreinigungen katalysieren die Nukleation von Keimen. An Korngrenzen verringert sich die aufzuwendende freie Energie

$$
\Delta G \sigma=\Delta G-\frac{\sigma_{\alpha \alpha}}{4} a i^{2 / 3}
$$

$\left(\sigma_{\alpha \alpha}=\right.$ Oberflächenspannung an der $\alpha \alpha-G r e n z-$ schicht). Die Nukleation an Verunreinigungen (In$\operatorname{dex}$ s) wird erleichtert, wenn die intermolekularen Kräfte zwischen $\beta$ und $\mathrm{s}$ größer sind als zwischen $\beta$ und $\alpha$, d. h. wenn für die Oberflächenspannung gilt

$$
W_{\mathrm{s}}=\sigma_{\beta \mathrm{s}}-\sigma_{\alpha \mathrm{s}}<\sigma_{\alpha \beta} .
$$

Die freie Energie ergibt sich zu

$$
\Delta G_{\mathrm{s}}=\Delta G+W_{\mathrm{s}} a_{\beta \mathrm{s}} i^{2 / 3}
$$

$\left(a_{\beta \mathrm{s}}\right.$ ist die Grenzfläche zwischen $\beta$ und $\mathrm{s}$ ).

Für die Nukleationsrate bei heterogener Nukleation läßt sich ein allgemeiner Ausdruck angeben:

$I=\sum_{j} \frac{N_{j}}{V} K_{j}{ }^{\prime} v_{0} \exp \left\{-\left(\Delta G_{\mathrm{s}}{ }^{*}+\Delta G_{\mathrm{a}}{ }^{\prime}\right) / k T\right\}$.

$N_{j} / V$ ist die Anzahl der Keimzentren pro Volumeneinheit. Der Frequenzfaktor $K_{j}^{\prime} v$ kann für jedes Keimzentrum verschieden groß sein. $\Delta G_{\mathrm{a}}{ }^{\prime}$, die Aktivierungsenergie für Korngrenzendiffusion, ist im allgemeinen kleiner als $\Delta G_{\mathrm{a}}$ in Gl. $\left(4^{\prime \prime}\right)$, d. h. die Diffusion über Korngrenzen geht schneller vor sich als die Volumendiffusion.

\section{Nukleation in mikroskopischen Hohlräumen}

Die Tieftemperaturphase $\beta$ kann als mikroskopischer Einschluß sehr stabil sein. Das System muß oft stark überhitzt werden $\left(\Delta T^{+}\right.$groß), um Überreste $\operatorname{der} \beta$-Phase in kleinen Hohlräumen zu schmelzen. Das Schmelzen (Index m, Index $f$ bezieht sich auf Gefrieren) findet statt, wenn

$$
\Delta G_{\mathrm{cav}}=r^{2} \pi h \Delta G_{\mathrm{v}}+2 r \pi h\left(\sigma_{\alpha \mathrm{s}}-\sigma_{\beta \mathrm{s}}\right)
$$

null wird. Der kritische Radius des mikroskopischen Hohlraums wird dann mit Gl. (5) (siehe Abb. 1)

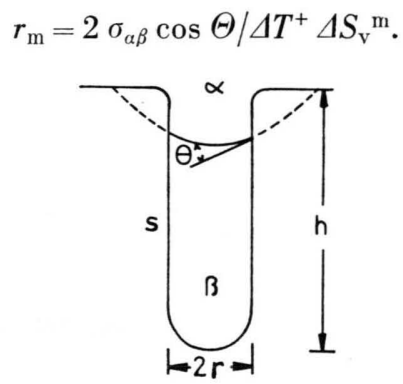

Abb. 1. Nukleation der $\beta$-Phase in einem zylindrischen Hohlraum.

Der Radius eines kritischen Keims läßt sich aus Gl. (7) berechnen

$$
r^{*}=2 \sigma_{\alpha \beta} \sin \Theta / \Delta T^{-} \Delta S_{\mathrm{v}}{ }^{\mathrm{f}} .
$$

Reste von $\beta$ in mikroskopischen Hohlräumen werden spontan weiterwachsen, wenn die Unterkühlung so groß ist, daß $r^{*}=r_{\mathrm{m}}$. Aus dieser Bedingung folgt, daß die zur Bildung eines kritischen Keims notwendige Unterkühlung proportional zur vorangegangenen Überhitzung ist. Erst für große $\Delta T^{-}$ wird die Nukleation unabhängig von der thermischen Vorgeschichte, weil dann die heterogene $\mathrm{Nu}$ kleation an Fehlstellen und Verunreinigungen überwiegt.

\section{Wachstum der überkritischen Keime}

Die Geschwindigkeit $u$, mit der die $\alpha \beta$-Phasengrenze eines überkritischen Keims in die $\alpha$-Phase hineinwächst, ist gegeben durch

$$
u=\lambda v_{0} e^{-\Delta G_{\mathrm{a}}{ }^{\prime} / k T}\left(1-e^{-v \Delta G_{\mathrm{v}} / k T}\right)
$$

(siehe Abb. 2), $\Delta G_{\mathrm{a}}{ }^{\prime}$ kann hier sowohl die Aktivierungsenergie für Volumendiffusion als auch für Diffusion über Korngrenzen sein. Führt man einen effektiven Diffusionskoeffizienten ein

$$
D=D_{0} e^{-\Delta G_{\mathrm{a}}{ }^{\prime} / k T} \cong v_{0} \lambda^{2} e^{-\Delta G_{\mathrm{a}^{\prime}} / k T},
$$




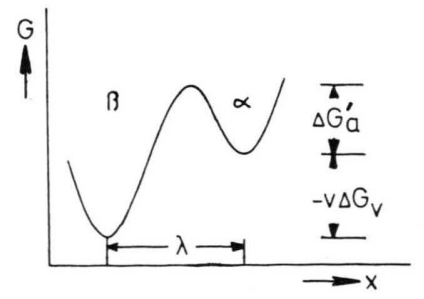

Abb. 2. Potentialkurve für ein Molekül, das die $\alpha-\beta$-Grenzschicht überschreitet.

so erhält man aus Gl. (12) und Gl. (5) für schwache Unterkühlung

$$
u \cong(D / \lambda) \Delta S_{\mathrm{v}} \Delta T^{-} / k T .
$$

Bei starker Unterkühlung wird die Geschwindigkeit $u$ unabhängig von $\Delta T^{-}$,

$$
u \cong D / \lambda .
$$

\section{Experimentelle Voraussetzungen}

Zur Bestimmung der elastischen Konstanten wurde die Ultraschallimpuls-Echo-Methode verwen$\operatorname{det}^{28}$. Aus der Laufzeit $\tau$ des Ultraschallimpulses (erzeugt durch einen 7-MHz-Quarzschwinger, XSchnitt) senkrecht zur $c$-Achse wurden die elastischen Konstanten für reines $\mathrm{D}_{2} \mathrm{O}\left(c_{33}\right)$ und für $\mathrm{HF}$ dotiertes $\mathrm{D}_{2} \mathrm{O}\left(c_{33 \mathrm{HF}}\right)$ ermittelt. Nach Mason ${ }^{29}$ ergibt sich $c_{33}$ aus folgender Gleichung

$$
c_{33}(T)=\varrho(T) \frac{l(T)^{2}}{\tau}=\varrho_{0} l_{0}^{2} / \alpha(T) \tau^{2}
$$

mit dem Ausdehnungskoeffizienten $\alpha(T)=\left(l_{0}-\right.$ $l(T)) / l_{0} ; l_{0}$ ist die Laufstrecke des Ultraschallimpulses bei $0{ }^{\circ} \mathrm{C}$. Für die Dichte von $\mathrm{D}_{2} \mathrm{O}$ wurde der Wert von UREY und TEAL ${ }^{30} \varrho_{0}=1,0402 \mathrm{~g} / \mathrm{cm}^{3}$ bei $0{ }^{\circ} \mathrm{C}$ verwendet. Für die HF-dotierten Eiskristalle wurde derselbe Wert eingesetzt; das ergibt einen Fehler im Absolutbetrag der elastischen Konstanten von 1,6\%. Der Ausdehnungskoeffizient $\alpha(T)$ ist für $\mathrm{H}_{2} \mathrm{O}$ - und $\mathrm{D}_{2} \mathrm{O}$-Eis gleich ${ }^{31}$. Der rechnerisch ermittelte Absolutfehler von $6 \%$ stimmt mit dem experimentell gefundenen überein.

Die Temperaturabhängigkeit der Laufzeit wurde mit Hilfe der Doppelimpuls-Interferenzmethode gemessen ${ }^{28}$. Überlagert man der ursprünglichen Im-

28 G. Freyer u. H. D. Tietz, Wiss. Z. Techn. Hochschule, Magdeburg 10, 117 [1966].

29 W. P. Mason, Physical Acoustics, D. van Norstrand, Princeton 1958, p. 371 . pulsreihe (eingestrahlter Impuls + reflektierte Impulse) eine zweite mit einer Verzögerungszeit $t$, die ein ganzes Vielfaches von $2 \tau$ ist, so kommt es, je nach Phasenlage der beiden Impulsreihen zueinan. der, zu einer Verstärkung oder Abschwächung der einzelnen empfangenen Signale. Die durch eine Temperaturänderung bedingte Änderung von $\tau$ bewirkt eine Phasenverschiebung, die durch Änderung von $t$ wieder kompensiert werden kann. Die Änderung von $t$ ist somit ein Maß für die Änderung der Laufzeit mit der Temperatur. Der Fehler bei dieser Relativmessung beträgt $0,3 \%$.

Für die Zucht der Einkristalle stand $\mathrm{D}_{2} \mathrm{O}$ zur Verfügung, das nach mehrmaliger Destillation eine Leitfähigkeit von $1,5-3,0 \mu \mathrm{S} / \mathrm{cm}$ und einen Deuterierungsgrad von 97\% hatte. Die Ausgangslösung für die dotierten Kristalle bestand aus einer Mischung von $\mathrm{D}_{2} \mathrm{O}$ (86\%), $\mathrm{H}_{2} \mathrm{O}(14 \%)$ und $\mathrm{HF}$ $(0,01 \%)$; die Leitfähigkeit betrug $800 \mu \mathrm{S} / \mathrm{cm}$.

Die Einkristalle wurden nach einer modifizierten Bridgman-Methode ${ }^{32}$ senkrecht zur $c$-Achse gezogen. Für die Messung wurden nur Kristallbereiche verwendet, die korngrenzenfrei waren. Die Präparation der Proben erfolgte in einem Kühlraum bei $-10^{\circ} \mathrm{C}$. Mit einem Konoskop wurde die Orientierung der Kristalle auf \pm 1 Bogengrad genau festgelegt. Die orientierten Kristalle wurden auf einer Drehbank zu einer zylindrischen Form mit einem Durchmesser von $16 \mathrm{~mm}$ und einer Länge von $30-35 \mathrm{~mm}$ abgedreht. Dabei mußte besonders auf Parallelität und Planheit der Endflächen geachtet werden, da sich schon kleine Keilwinkel und Unebenheiten störend auf die reflektierten Impulse auswirken. Vor dem endgültigen Einbau der Probe in den Probenbehälter wurde die Länge mit einer Mikrometerschraube gemessen. Als Kopplungssubstanz zwischen Schwinger und Probe hat sich im Temperaturbereich zwischen $80^{\circ} \mathrm{K}$ und $200^{\circ} \mathrm{K} 4$ Methyl-1 Penten gut bewährt ${ }^{17}$.

Sowohl mit reinen als auch mit HF-dotierten Proben wurden insgesamt drei verschiedene Meßreihen durchgeführt:

1. Die Proben wurden im Bereich zwischen $80{ }^{\circ} \mathrm{K}$ und $200{ }^{\circ} \mathrm{K}$ langsam mit einer Geschwindigkeit von $1 \mathrm{grd} / \mathrm{min}$ abgekühlt, bzw. aufgeheizt.

30 H. C. Urey u. G. K. Teal, Rev. Mod. Phys. 7, 34 [1935].

31 G. Dantl, Z. Phys. 166, 115 [1962] ; 169, 466 [1962].

32 H. Engelhardt, H. Müller-KrumbhaAr, B. Bullemer u. N. RieHL, J. Appl. Phys. 40, 5308 [1969]. 
2. Zwischen $100{ }^{\circ} \mathrm{K}$ und $150^{\circ} \mathrm{K}$ wurden die Proben bei verschiedenen Temperaturen jeweils etwa 30 Minuten getempert.

3. In einer dritten Versuchsreihe wurde ein Temperaturzyklus mehrere Male durchfahren, wobei die Proben an den Umkehrpunkten $T_{1}$ und $T_{2}$ jeweils 15-20 Minuten getempert wurden. Als Umkehrpunkte wurden $T_{1}=130{ }^{\circ} \mathrm{K}$ und $T_{2}=$ $105^{\circ} \mathrm{K}$, bzw. $T_{1}=150{ }^{\circ} \mathrm{K}$ und $T_{2}=120^{\circ} \mathrm{K}$ gewählt.

\section{Ergebnisse}

Die Ergebnisse der in Abschnitt III beschriebenen drei Versuchsreihen an reinen und HF-dotierten $\mathrm{D}_{2} \mathrm{O}$-Eis-Einkristallen sind in Tab. 1 zusammengestellt. Zum Vergleich sind frühere Ergebnisse von $\mathrm{H}_{2} \mathrm{O}$-Eis ${ }^{17}$ mit aufgeführt.

\begin{tabular}{|c|c|c|c|}
\hline $\begin{array}{l}\text { Hinweis auf Ordnungs- } \\
\text { Unordnungs-Ưbergang bei }\end{array}$ & $\begin{array}{l}\mathrm{D}_{2} \mathrm{O} \text {-Eis } \\
\text { rein }\end{array}$ & $\begin{array}{l}\mathrm{D}_{2} \mathrm{O} \text {-Eis } \\
\mathrm{HF} \text {-dot. }\end{array}$ & $\begin{array}{l}\mathrm{H}_{2} \mathrm{O} \text {-Eis } \\
\text { rein }\end{array}$ \\
\hline $\begin{array}{l}\text { Versuchsreihe } 1 \\
\text { langsame Temperatur- } \\
\text { änderung }\end{array}$ & nein & nein & ja $(-)$ \\
\hline $\begin{array}{l}\text { Versuchsreihe } 2 \\
\text { Tempern }\end{array}$ & nein & ja $(+)$ & ja $(-)$ \\
\hline $\begin{array}{l}\text { Versuchsreihe } 3 \\
\text { Temperaturzyklus }\end{array}$ & nein & $\mathrm{ja}(+)$ & \\
\hline $\begin{array}{l}\text { Zeitdauer für Einstellung } \\
\text { des Gleichgewichts }\end{array}$ & & $30 \mathrm{~min}$ & $30 \mathrm{~min}$ \\
\hline Größe des Effekts & & $1-1,4 \%$ & $0,6-1,2 \%$ \\
\hline $\begin{array}{l}\text { Oberste Grenztemperatur, } \\
\text { bei der Anomalie auftritt }\end{array}$ & & $160^{\circ} \mathrm{K}$ & $150^{\circ} \mathrm{K}$ \\
\hline
\end{tabular}

Tab. 1. Ergebnisse der Ultraschalluntersuchungen an reinem und $\mathrm{HF}$-dotiertem $\mathrm{D}_{2} \mathrm{O}$-Eis und an $\mathrm{H}_{2} \mathrm{O}$-Eis ${ }^{34}$. (+) Kristall wird härter, (-) Kristall wird weicher.

Beim Aufheizen, bzw. Abkühlen der reinen und $\mathrm{HF}$-dotierten $\mathrm{D}_{2} \mathrm{O}$-Kristalle mit einer Geschwindigkeit von $1 \mathrm{grd} / \mathrm{min}$ konnte im Bereich zwischen $80^{\circ} \mathrm{K}$ und $200{ }^{\circ} \mathrm{K}$ kein anomales Verhalten der elastischen Konstanten festgestellt werden. Die elastischen Konstanten $c_{33}$ und $c_{33 \mathrm{HF}}$ zeigten eine parabelförmige Abhängigkeit von der Temperatur wie sie schon von MiTZDORF ${ }^{23,}{ }^{24}$ gemessen wurde und wie es auch nach der Theorie von BoRN ${ }^{33}$ sein

33 M. Born, J. Chem. Phys. 7, 591 [1939].

34 G. DantL, Phys. kondens. Materie 7, 390 [1968].

35 T. M. Proctor JR., J. Acoust. Soc. Amer. 39, 972 [1966]. soll. Auf einen Vergleich der Absolutwerte von $c_{33}$ und $c_{33 \mathrm{HF}}$ untereinander, sowie mit den früher gemessenen $\mathrm{D}_{2} \mathrm{O}$-Werten ${ }^{24}$ und den $\mathrm{H}_{2} \mathrm{O}$-Werten ${ }^{34,}{ }^{35}$ soll hier nicht eingegangen werden. Eine ausführliche Diskussion ist in ${ }^{36} \mathrm{zu}$ finden (siehe in diesem Zusammenhang auch ${ }^{34,37}$ ).

30-minütiges Tempern der reinen und HF-dotierten $\mathrm{D}_{2} \mathrm{O}$-Kristalle im Bereich zwischen $100{ }^{\circ} \mathrm{K}$ und $150{ }^{\circ} \mathrm{K}$ brachte nur bei den HF-dotierten Kristallen eine Erhöhung der elastischen Konstanten um etwa 0,3\%. Diese Änderung von $c_{33 \mathrm{HF}}$ liegt zwar noch im Bereich der Fehlergrenze, aber die Tendenz einer Zunahme von $c_{33 \mathrm{HF}}$ ist bei diesem einfachen Temperversuch schon klar ersichtlich: die Abweichungen bei den einzelnen Messungen summieren sich auf, d.h., der nach der 3. Messung erreichte Absolutwert liegt um etwa $1 \%$ höher als der Wert, den man durch Extrapolation vom Anfangswert der 1. Messung auf die entsprechende Temperatur der 3. Messung erhalten würde (siehe Abb. 3).

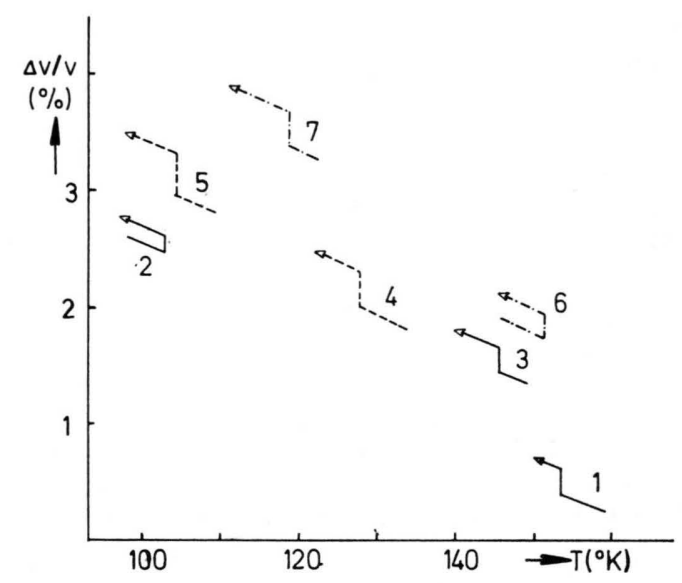

Abb. 3. Änderung der Schallgeschwindigkeit $v=\left(c_{33 \mathrm{HF}} / \varrho\right)^{1 / 2}$ bei jeweils 30-minütiger Temperung. Zwischen den Messungen 3 und 4 sowie 5 und 6 wurde der Kristall jeweils 12 Stunden in Stickstoff gelagert. Die Pfeile geben die Richtung der Temperaturänderung an.

Nach mehrmaligem Durchlaufen der in Abschnitt III beschriebenen Temperaturzyklen zeigten sich bei reinem $\mathrm{D}_{2} \mathrm{O}$-Eis wiederum keine Anzeichen für ein anomales Verhalten. Bei HF-dotiertem $\mathrm{D}_{2} \mathrm{O}$ Eis nahm die elastische Konstante im Lauf der Messungen zu. Zweimaliges Durchlaufen des Zyklus

36 I. Sesselmann, Diplomarbeit, Techn. Universität München 1970.

37 G. DantL, Polarforschung VI/37, 129 [1969]. - G. DantL u. I. Gregora, Naturwiss. 55, 176 [1968]. 
zwischen $105^{\circ} \mathrm{K}$ und $130{ }^{\circ} \mathrm{K}$ ergab eine Erhöhung von $c_{33 \mathrm{HF}}$ um $1,4 \%$. Durch anschließendes dreimaliges Durchlaufen des Zyklus zwischne $120^{\circ} \mathrm{K}$ und $150{ }^{\circ} \mathrm{K}$ erhöhte sich der Absolutwert von $c_{33 \mathrm{HF}}$ nochmals um $1 \%$ (siehe Abb. 4). Zwischen den beiden Zyklen wurde der Kristall bei $\mathrm{N}_{2}$-Temperatur gelagert.

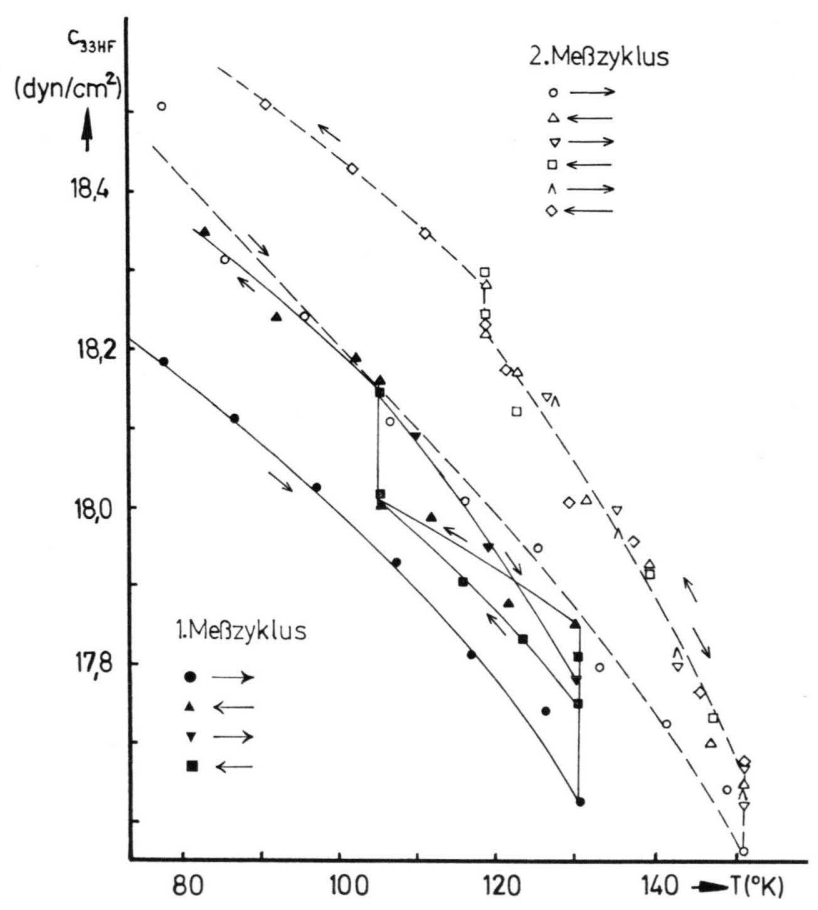

Abb. 4. Abweichungen der elastischen Konstanten $c_{33} \mathrm{HF}$ beim zyklischen Meßverfahren. Zwischen 1. und 2. Meßzyklus wurde der Kristall 12 Stunden in Stickstoff gelagert. Die Pfeile geben die Richtung der Temperaturänderung an.

\section{Diskussion der Ergebnisse}

Aus den in Tab. 1 aufgeführten Ergebnissen können folgende Erkenntnisse gewonnen werden:

1. Ein Ordnungs-Unordnungs-Übergang in der Protonenstruktur konnte bei reinem $\mathrm{D}_{2} \mathrm{O}$-Eis nicht nachgewiesen werden.

2. Verunreinigungen und Kristallfehler begünstigen den Ordnungs-Unordnungs-Übergang.

3. Die Größe der gemessenen Anomalie ist abhängig von der thermischen Vorgeschichte.

4. HF-dotiertes schweres Eis zeigt im Gegensatz zum reinen leichten Eis eine positive Anomalie der elastischen Konstanten. HF-dotiertes $\mathrm{D}_{2} \mathrm{O}$ -

Eis wird härter, reines $\mathrm{H}_{2} \mathrm{O}$-Eis weicher.

Diese experimentellen Befunde lassen sich durch folgende Vorstellungen erklären:

Homogene Nukleation wird in den hier verwendeten reinen Kristallen eine untergeordnete Rolle spielen. „Rein“ bedeutet in diesem Fall, daß die Kristalle nicht bewußt verunreinigt wurden. Reines Eis hat normalerweise eine Versetzungsdichte von $10^{2}-10^{4}$ Versetzungen $/ \mathrm{cm}^{2}$, die Leitfähigkeit des Schmelzwassers liegt um zwei Größenordnungen über dem theoretischen Wert für ideal reines Wasser $\left(4,41 \cdot 10^{-8} \Omega^{-1} \mathrm{~cm}^{-1}\right.$ bei $\left.18{ }^{\circ} \mathrm{C}\right)$. Ideales Eis kann prinzipiell nicht hergestellt werden. Im realen Eis sind noch genügend Fehlstellen chemischer und struktureller Art vorhanden, um heterogene Nukleation (zumindest im $\mathrm{H}_{2} \mathrm{O}$-Eis) zu ermöglichen. Nach Gl. (8) muß der Frequenzfaktor $K_{j}^{\prime} v$ dafür verantwortlich gemacht werden, $\mathrm{da} \beta$ beim reinen $\mathrm{D}_{2} \mathrm{O}$-Eis keine Anomalie auftritt. Eine grobe $\mathrm{Ab}$ schätzung der Nukleationsraten (die Anzahl der Keimzentren $N_{j} / V$ wird für leichtes und schweres Eis als gleich angenommen) nach Gl. (8) mit Gl. (4') und Gl. (4") ergibt für leichtes Eis eine etwa doppelt so große Nukleationsrate wie für schweres Eis.

Durch das von WORKMAN und REYNOLDS ${ }^{38}$ nachgewiesene Wachstumspotential in der Größenordnung $100 \mathrm{~V}$ entstehen während der Kristallisation polare Bereiche. Die polare Struktur wird innerhalb kurzer Zeit wieder abgebaut; diese Zeit ist abhängig von der Lagertemperatur und beträgt bei $-3{ }^{\circ} \mathrm{C}$ einige Stunden ${ }^{17}$. Mit diesem Alterungsprozeß erklärt DANTL ${ }^{34,37}$ die unterschiedlichen Ergebnisse beim Piezoeffekt, sowie bei Messungen der Dichte und der elastischen Konstanten. Die Werte für die Dichte und die elastischen Konstanten nehmen durch Alterung ab. Allerdings liegt die experimentell gefundene geringste Dichte immer noch um $4 \cdot 10^{-4}$ über dem Wert, der sich aus den röntgenographischen Daten ergibt ${ }^{19}$ (die Meßgenauigkeit bei den Dichtemessungen betrug $4 \cdot 10^{-5}$ ). Das deutet darauf hin, daß mikroskopische polare Bereiche auch bei extremer Temperung noch bestehen bleiben. Nach Gl. (10) ist eine, von der Größe $r_{\mathrm{m}}$ dieser Bereiche abhängige Überhitzung $\Delta T^{+}$notwendig, um die Bereiche zu zerstören. Die Überhitzung kann dabei so groß werden, daß der Schmelzpunkt des Eises über-

38 E. J. Workman u. S. E. Reynolds, Phys. Rev. 78, 254 [1950]. 
schritten werden muß, um auch die polaren Bereiche aufzuschmelzen.

Diese nicht ausheilbaren polaren Bereiche werden nun verantwortlich gemacht für das unterschiedliche Verhalten von reinem und HF-dotiertem Eis. Durch den Einbau von Verunreinigungen erhöht sich die Anzahl der Strukturfehler ${ }^{39}$. Es werden also, im Vergleich zu reinem Eis, mehr und größere mikroskopische Hohlräume zur Verfügung stehen, in denen der polarisiente Zustand bei Überhitzung stabil bleibt. Ist der polarisierte Bereich so groß, daß bei einer bestimmten Unterkühlung sein Radius $r_{\mathrm{m}}$ gleich dem Radius des kritischen Keims $r^{*}$ wird, dann kann dieser nun kritische Keim spontan weiterwachsen. Dieser spontane Übergang von $\operatorname{der} \alpha$ - in die $\beta$-Phase macht sich in einer Erhöhung der elastischen Konstanten bemerkbar (Abb. 5 a). Das bei reinem $\mathrm{H}_{2} \mathrm{O}$-Eis beobachtete Aufweichen am Ubergangspunkt ${ }^{17}$ deutet auf Fluktuationen hin. Im reinen Eis sind zwar nach der Zucht polarisierte Bereiche vorhanden, ihre Größe ist jedoch unterkritisch.

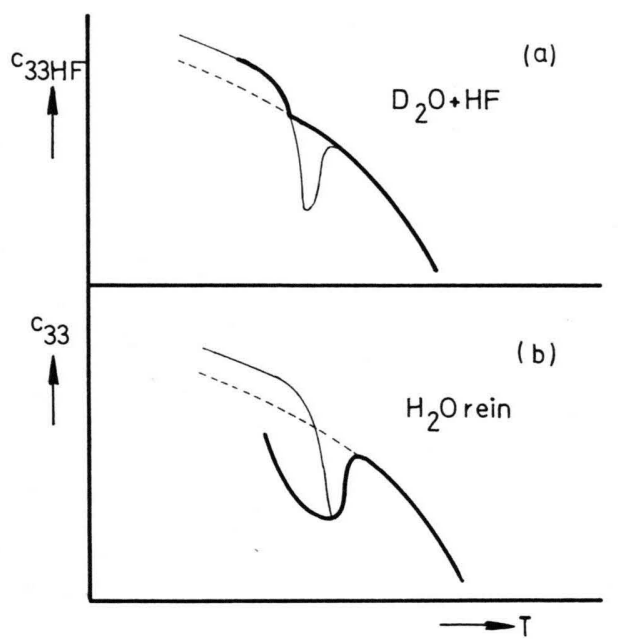

Abb. 5. Schematischer Verlauf der elastischen Konstanten beim Ordnungs-Unordnungs-Übergang: a) $c_{33 \mathrm{HF}}$ für HF-dotiertes $\mathrm{D}_{2} \mathrm{O}$-Eis, b) $c_{33}$ für reines $\mathrm{H}_{2} \mathrm{O}$-Eis (dick ausgezogene $\mathrm{Li}$ nien). Gestrichelt ist der Verlauf bei normalem Verhalten, ohne Phasenübergang; dünn durchgezogen der erwartete Verlauf bei Nukleation und Wachstum $\operatorname{der} \beta$-Phase.
Die kritischen Keime entstehen überwiegend durch Nukleation. Das Wachstum der kritischen Keime friert bei Temperaturerniedrigung allmählich ein. Der in Abb. 5 b dargestellte typische Verlauf der elastischen Konstanten für den Fall heterogener Nukleation wurde auch im Experiment gefunden ${ }^{17}$.

Der weite Temperaturbereich, in dem elastische Anomalien beim HF-dotierten $\mathrm{D}_{2} \mathrm{O}$-Eis gemessen wurden, ergibt sich aus der unterschiedlichen Größe der einzelnen polarisierten Mikrobereiche. Bei großen Bereichen wird nur geringe Unterkühlung benötigt [Gl. (11)], je kleiner die Bereiche sind, desto größer muß die Unterkühlung sein. Die im Abschnitt IV beschriebene Aufsummation der Abweichungen bei aufeinanderfolgenden Temperungen bei verschiedenen Temperaturen kann dadurch erklärt werden, daß noch unterkritische Keime vorhanden sind, die durch heterogene Nukleation kritisch werden. Die Möglichkeit, daß alle Keime zwar kritisch sind, aber bei einer Temperatur $T<T_{\mathrm{c}}$ weiterwachsen, kann nicht ausgeschlossen werden.

Die oben beschriebenen Vorstellungen für den Ordnungs-Unordnungs-Übergang können die bisherigen Ergebnisse der Ultraschall-Untersuchungen an reinem und HF-dotiertem Eis erklären. Eine Prüfung der Ideen ist möglich, wenn die an HF-dotiertem schwerem Eis gemachten Messungen an $\mathrm{HF}$. dotiertem leichtem Eis wiederholt werden. Untersuchungen an pulverisiertem Eis mit der Korngröße als Parameter sollten weiterhin zur Klärung des Mechanismus beitragen. Wenn die Eiskörner kleiner werden als die stabilen polarisierten Bereiche, sollte kein Ordnungs-Unordnungs-Übergang mehr stattfinden.

Unser herzlicher Dank gilt unserem verehrten Lehrer, Herrn Prof. Dr. N. RIEHL, der in uns das Interesse an der Eisphysik erweckte. Frl. U. Mitzdorf danken wir für Anregungen und klärende Diskussionen. Die Sachmittel wurden von der Stiftung Volkswagenwerk bereitgestellt.

39 F. K. TRUBy, Science 121, 104 [1955]. 